\title{
ABSTRACTS OF PAPERS
}

\section{SUBMITTED FOR PRESENTATION TO THE SOCIETY}

The following papers have been submitted to the Secretary and the Associate Secretaries of the Society for presentation at meetings of the Society. They are numbered serially throughout this volume. Cross-references to them in the reports of the meetings will give the number of this volume, the number of this issue, and the serial number of the abstract.

104. Mr. Garrett Birkhoff: Combinatorial relations in finite projective geometries.

Under the restriction of finiteness, it is shown that $B$-lattices satisfying a (non-unique) complementarity relation are equivalent to direct joins of Boolean algebras and projective geometries. This has interpretations in projective geometry and group theory. (Received January 24, 1934.)

105. Mr. Aaron Fialkow: General theorems on trajectories and lines of force.

We generalize certain theorems of Kasner relative to the geometry of arbitrary fields of force (Science, June 24, 1932; p. 671). Consider the motion of a particle which starts from rest at a point where the line of force has contact of the $n$th order with its tangent. Kasner has shown that the trajectory has the same contact and that the ratio of the infinitesimal departures of the line of force and the trajectory from their common tangent is $2 n+1$. We extend these results to the more general cases in which the contact between the line of force and its tangent is of any order, finite (integral, fractional or irrational) or infinite, as well as to cases in which no definite order of contact exists. In general, the ratio of departures approaches all values within a closed interval (rather than a single point), the interval varying with the order of contact. We also show that the limiting values of the ratio are uniquely determined by the direction of the force along any segment of the tangent line which includes the initial point. Kasner's theorems for the cases of non-zero speed and motion in a resisting medium are also generalized. (Received January 15, 1934.)

106. Professor E. V. Huntington: Independent postulates related to C. I. Lewis's theory of strict implication.

This paper presents an abstract mathematical system of which Lewis's system (as verbally formulated) may be regarded as an instance. Base: a class $K$ (interpretable as "propositions"); two subclasses $Q$ and $D$ (interpretable as "false" and "necessarily false"); $a \times b$ (interpretable as " $a$ and $b$ "); $a^{\prime}$ (interpretable as the "contradictory" of $a$ ); and $a=b$, meaning that $a$ and $b$ are interchangeable for the purposes of the system. Definitions: (I) ( $a$ in $P$ ) means ( $a$ in $K$ and $a$ not in $Q$ ). (II) ( $a$ in $A)$ means $\left(a^{\prime}\right.$ in $\left.D\right)$. (III) $(a<b)$ means $\left(a b^{\prime}\right.$ in 
$D$ ). Postulates: (1) If $a$ in $K$ and $b$ in $K$, then $a b$ in $K$. (2) If $a$ in $K$, then $a^{\prime}$ in $K$. (3) $a b=b a$. (4) $(a b) c=a(b c)$. (5) $\left(a^{\prime} b^{\prime}\right)^{\prime}\left(a^{\prime} b\right)^{\prime}=a$. (6) If $a$ in $Q$, then $a$ in $K$. (7) If $a$ in $D$, then $a$ on $Q$. (8) If $a$ in $D$ and $b$ in $K$, then $a b$ in $D$. (9) If $a$ in $P$ and $b$ in $P$, then $a b$ in $P$. (10) If $a$ in $K$ and $a^{\prime}$ in $Q$, then $a$ in $P$. (11) If $a$ in $A$ and $b$ in $A$, then $a b$ in $A$. (12) There exists at least one element $b$ in $K$ such that $b$ not in $D$ and $B$ not in $A$. (13, optional) If $a$ in $D$ and $b$ in $D$, then $a=b$. (When (13) is added, (11) becomes redundant.) Points of divergence between ordinary mathematical methods and certain procedures characteristic of the logistic method are indicated. (Received January 29, 1934.)

107. Professor Louis Weisner: Criteria for the irreducibility of polynomials.

Let $A(x)$ be a polynomial of degree $n$ with integral coefficients, the coefficient of $x^{k}$ being $a_{n-k}$, and let $a_{n}= \pm k p^{m}$, where $p$ is a prime which does not divide $a_{n-1}$ if $m>1$. Let $L$ and $M$ be lower and upper bounds of the absolute values of the roots of $A(x)=0$. The author proves that if $A(x)$ is reducible in the field of rational numbers and $L \geqq 1$, then $k \geqq L$, while if $M \geqq 1$, then $p^{m} \leqq\left|a_{0}\right| M^{n-1}$. It is possible to construct large classes of polynomials which satisfy the hypothesis, but which violate one of the inequalities of the conclusion, and which are therefore irreducible. Further, by effecting a linear transformation on the polynomial, a theorem is deduced which may enable one to infer the irreducibility of a polynomial from its table of values. (Received December $30,1933$.

\section{Professor Antoni Zygmund: Some points in the theory of} trigonometric and power series.

This paper consists of six notes treating various related topics. In the first note it is proved that if the partial sums of the Fourier series of $f(x)$ are bounded below by a negative integrable function, then almost everywhere $f(x)$ is equal to the arithmetic mean of lim sup $s_{n}$ and lim inf $s_{n}$. The second note is concerned with the exponent of convergence of the sequence of Fourier coefficients of a function which is of bounded variation and satisfies a Lipschitz condition of positive order. In the third note there is found a simple proof of a theorem of Kolmogoroff concerning the conjugate of a Fourier series. The fourth note contains a new proof of the classical theorem of Fejér-F. Riesz and of some of its extensions. The fifth note deals with the extension of some results of Hardy and Littlewood concerning the theory of fractional integrals, while the sixth note contains extensions of some results of the same authors in the theory of Fourier constants. (Received January 24, 1934.)

\section{Professor E. P. Lane: The moving trihedron.}

A classical method of studying the metric differential geometry of curves and surfaces in three-dimensional space is based upon the use of a moving trihedron. Professor G. A. Bliss constructed several years ago a theory of the moving trihedron in the theory of curves, and used it in his lectures, but has never published it. He used the moving trihedron whose edges are the tangent, principal normal, and binormal at a point of a curve, and based his theory on certain recursion formulas, which are the novel feature of the treatment. They 
give the components, in the moving coordinate system, of the derivatives of any order of the vector defining a curve whose points are in one-to-one correspondence with the points of the given curve. The present paper outlines the theory of curves and then extends it to surfaces. The author uses the moving trihedron whose edges are the tangents of the lines of curvature and the normal at a point of a surface. Recursion formulas are again employed. The six fundamental coefficients of a surface, whose points are in one-to-one correspondence with those of a given surface, are calculated in terms of the components of the derivatives of the vector defining the former surface, referred to the moving trihedron of the latter. The results are capable of wide application. (Received January 8, 1934.)

\section{Professor H. R. Brahana: Irreducible quartic congruences.}

The irreducible quartics belonging to the modular field determined by a prime $p$ constitute $(p-1) / 2$ sets of conjugates under the group of linear fractional transformations with coefficients also in the modular field. Each set is characterized by the cross ratio of the roots of the quartic; each set is also characterized by the value of the absolute invariant of the quartic. (Received January 19, 1934.)

111. Professor R. D. Carmichael: A general expansion theorem with applications to certain linear integral equations of infinite order.

A general theorem is given for the expansion of functions $f(x)$, analytic at $x=0$, in the form $f(x)=\sum_{\nu=0}^{\infty} c_{\nu}\left\{x^{\nu} / \lambda_{0} \lambda_{1} \cdots \lambda_{\nu}\right\}\left\{1+a_{1}(x) \cdot x / \lambda_{\nu+1}+a_{2}(x)\right.$ $\left.\cdot x^{2} /\left(\lambda_{\nu+1} \lambda_{\nu+2}\right)+\cdots\right\}$, where $\lambda_{0}, \lambda_{1}, \cdots$ is a given non-decreasing sequence of positive constants with the limit infinity and where $a_{1}(x), a_{2}(x), \cdots$ is a sequence of given analytic functions subject to suitable restrictions. The expansion theorem is applied in the almost immediate derivation of existence theorems for linear differential equations, for certain linear integral equations of infinite order, and for certain integro-differential equations. (Received January 27, 1934.)

112. Professors Tibor Radó and Lincoln LaPaz: On a converse of the Kneser transversality theorem.

In the special case in which transversality is orthogonality, E. Kasner for euclidean 3-space (Princeton Colloquium Lectures, 1909, p. 47) and J. Lipke for Riemannian $n$-space (Transactions of this Society, vol. 13 (1912), p. 77) have proved an important converse of the Kneser transversality theorem. J. A. Schouten (Nieuw Archief voor Wiskunde (1927), p. 97) has given a simplified proof of Lipke's results. Independently W. Blaschke (Nieuw Archief voor Wiskunde (1927), p. 202) and J. Douglas (Transactions of this Society, vol. 29 (1927), p. 401) have given proofs of a converse of Kneser's theorem for the case of a general transversality. The purpose of the present paper is to give a proof which emphasizes the very simple geometrical background of the theorem in question. The proof is based on a congruence relation obtained by applying to finite arcs a construction employed by Blaschke for infinitesimal arcs. (Received February 6, 1934.) 
113. Professor T. R. Hollcroft: Webs of quadric hypersurfaces in $r$ dimensions.

The properties of the web of quadric hypersurfaces in $S_{r}$ are obtained by means of the $(1,1)$ correspondence existing between the quadric hypersurfaces of the web and the planes of an $S_{3}$. For $r \geqq 4$, the web contains no completely degenerate quadrics. For all values of $r$, however, the web contains $r(r+1)(r+2)$ pencils of quadric hypersurfaces that have a line in common. (Received March 9, 1934.)

114. Professor Virgil Snyder and Dr. J. M. Clarkson: $A n$ involutorial line transformation determined by a bilinear congruence of twisted elliptic quartics.

Given a fixed plane $\gamma$ and two non-singular pencils of quadric surfaces $H_{1}-\alpha H_{2}=0, K_{1}-\beta K_{2}=0$, having as basis curves the twisted quartics $\alpha, \beta$ respectively. The curve $C_{4}(\alpha, \beta)$ of intersection of one quadric of each pencil is determined when $\alpha, \beta$ are given. As the parameters $\alpha, \beta$ vary independently, $C_{4}(\alpha, \beta)$ describes a bilinear congruence of twisted quartics of first species. An arbitrary line $(y)$ of space is bisecant to just one $C_{4}$ of the congruence and meets $\gamma$ in a point $P$. Through $P$ there exists one other bisecant $(x)$ of the same $C_{4}$. The line transformation $(y) \sim(x)$ is discussed. (Received March 2, 1934.)

\section{Dr. A. B. Brown and Mr. Morris Halperin: On certain area-preserving maps.}

It is proved that any simply-connected region of finite area in the plane can be mapped in a continuous one-to-one manner with preservation of area on a circle-interior. A characterization is given in terms of a set of nested curves and a curve crossing them, which are mapped on concentric circles and a radius respectively. This covers among others all maps which when expressed in cartesian coordinates are given by functions having continuous first partial derivatives. Some of the results are extended to curved surfaces and threedimensional regions, with equations of the transformations given. The method permits extending the results to regions with infinite content. (Received February $7,1934$.

116. Dr. S. F. Barber (National Research Fellow) and Dr. Jacob Yerushalmy: Exceptional curves of the first kind. Preliminary report.

Heretofore in the theory of birational transformations, the cases of irreducible exceptional curves or those with at most two components have been considered. This paper treats the general type of exceptional curve, $L^{(1)}=\sum_{i=1}^{s} k_{i}^{(1)} L_{i}, k_{i}^{(1)} \geqq 1$. Associated with $L^{(1)}$ are found other exceptional curves $L^{(r)}=\sum_{i=r}^{s} k_{i}(r) L_{i}$. In the correspondence between $L^{(i)}$ and $O_{i}$, the position of these points is described by a branch of minimum order passing through them. It has been found that the branch of minimum order passes through $O_{i}(i=1, \cdots, t)$ with multiplicity $k_{t}^{(i)}, t=1, \cdots, s$. The authors are now 
investigating the connection with the characteristic exponents in the Puiseux expansion and the relation of the intersection numbers of the components with satellite and free points. (Received March 9, 1934.)

117. Dr. L. M. Blumenthal (National Research Fellow): The metric characterization of the $n$-dimensional spherical space.

The $n$-dimensional spherical space, $S_{n_{r} r}$, of radius $r$, is the surface of an $(n+1)$-dimensional sphere in a euclidean space of $n+1$ dimensions, with the distance between two points defined as the geodesic distance. This paper defines an abstract space $S_{r}$ as a metric space the elements of which satisfy the following two postulates: Postulate 1 . The sum of the three distances determined by any three points of the space does not exceed $2 \pi r, r>0$. Postulate 2 . If a quadruple of points of the space contains a triple that is isometric with three points of $S_{1, r}$, the quadruple can be isometrically imbedded in $S_{2, r}$. The space is said to be diametrized if for each point $p$ the space contains a point $\bar{p}$ such that $\bar{p} \bar{p}=\pi r$. This paper investigates the properties of $S_{r}$. The principal results obtained are stated in the two following theorems. Theorem 1. If $S_{r}$ is convex, complete, and diametrized, then any $n+1$ points of $S_{r}$ can be isometrically imbedded in $S_{n, r}$. Theorem 2 . If $S_{r}$ is convex, complete, separable, and diametrized, then $S_{r}$ may be transformed by an isometric transformation into $S_{n, r}$ or $H_{r}$, where $H_{r}$ is the spherical analogue of Hilbert space. (Received March 5, 1934.)

118. Dr. D. S. Nathan (National Research Fellow): Oneparameter groups of transformations in abstract vector spaces.

A transformation $T$ in a complete linear metric space $\mathfrak{B}$ satisfying the inequality $\|T f-T g\| \leqq C\|f-g\|, C \geqq 0$, for every pair of elements $f$ and $g$ in $\mathfrak{B}$, generates a one-parameter continuous group of transformations in $\mathfrak{B}$. When $T$ is a bounded linear transformation, the generated group is the group of bounded linear transformations $e^{t T},-\infty<t<\infty$. Conversely, a subset, in a certain neighborhood of the identity transformation, of a given one-parameter continuous group of bounded linear transformations $A_{t}$ in $\mathfrak{B}$ with the law of composition $A_{t_{1}} A_{t_{2}}=A_{t_{1}+t_{2}}$ is generated by the bounded linear transformation $\log \left(A_{t} / t\right)$, defined in $\mathfrak{B}$. (Received March 8,1934 .)

119. Mr. George Comenetz: Families of $\infty^{3}$ conics which are curvature trajectories.

Curvature trajectories are a special type of family of $\infty^{3}$ plane curves, constructed according to the following geometrical rule. We take a family of $\infty^{2}$ curves arbitrarily as a base. A curvature trajectory of these is a curve drawn through the base family in such a way as to have its curvature at each point equal to $c$ times the curvature of the member of the base family which it touches at that point, $c$ remaining constant on any one trajectory. This construction is projectively invariant. We show that the only families of curvature trajectories which consist solely of conics are the following. (A) The projections, from a fixed center on to a fixed plane, of all the conics drawn on a quadric surface. (B) The two types of families of conics which are dynamical 
trajectories, determined by Darboux: conics with a common focus, of the Newtonian field, with their projections; and conics with a common center, of the elastic field, with their projections. (See Kasner, Dynamical trajectories and curvature trajectories, to appear shortly in this Bulletin.) (Received March 8, 1934.)

120. Professor Edward Kasner: Mixed groups connected with polygons.

This paper generalizes a theory of mid-point polygons and central symmetry published in the American Mathematical Monthly, 1903. Centroidal polygons are introduced and multi-point transformations present themselves. The generalized symmetries generate a mixed group of $r \infty^{2(r-1)}$ transformations operating on sets of $r-1$ points. A joint paper with several of the author's students will appear in Scripta Mathematica. (Received March 5, 1934.)

\section{Professor R. L. Wilder: Generalized closed manifolds in n-space.}

We define a generalized closed $n$-dimensional manifold (=g.c. $n$-m.) as an $n$-dimensional compact metric space $M$ such that $(1) p^{n}(M)=1$, but for any proper closed subset $F$ of $M, p^{n}(F)=0$; (2) given $P \subset M$, there is an $\epsilon>0$ such that if $\gamma^{i} \subset S(P, \epsilon), 1 \leqq i \leqq n-1$, then $\gamma^{i} \sim 0$ in $M$; (3) given $P \subset M$ and $\epsilon>0$, there exist positive numbers $\delta$ and $\eta, \epsilon>\delta>\eta$, such that if $\gamma^{i}(0 \leqq i \leqq n-2)$ is a cycle of $F(P, \delta)$, then $\gamma^{i \sim 0}$ in $S(P, \epsilon)-S(P, \eta)$; and if $\gamma^{n-1}$ is a cycle of $F(P, \delta)$, then $\gamma^{n-1} \sim 0$ in $M-S(P, \eta)$. It is shown that in order that a set $M$ in $E_{n}$ should be a g.c. $(n-1)-\mathrm{m}$., it is necessary and sufficient that it be the boundary of a bounded, simply $(n-1)$-connected, uniformly locally $i$-connected $(=\mathrm{u} .1$. $i$-c.) domain $(0 \leqq i \leqq n-2)$. This condition may be replaced by the condition that $M$ be the common boundary of two u.1. 0-c. domains $D_{1}$ and $D_{2}$ one of which is u.l. $i$-c. for $1 \leqq i \leqq n-3$ and $p^{n-2}\left(E_{n}-M\right)$ finite. A new duality is established: $p^{i}\left(D_{1}\right)=p^{n-i-1}\left(D_{2}\right), 1 \leqq i \leqq n-2$, and it follows that $M$ satisfies the Poincaré duality relation. $M$ itself is locally $i$-connected for $0 \leqq i \leqq n-2$, and all its Betti numbers are finite. (In $E_{3}, M$ is a closed manifold in the classical sense.) If $M$ is a set satisfying only conditions (2) and (3) (with $n$ replaced by $n-1$ ), then its complementary domains all have boundaries that are g.c. $(n-1)$-m.'s. (Received March 9, 1934.)

122. Mr. Saunders MacLane: A systematic theory of mathematical symbolism.

The development of the Hilbert "proof theory" and of the "semantic" logic of the Polish school have emphasized the importance of the analysis of mathematical symbolism. This analysis should rank as one of the basic disciplines in the foundations of mathematics on a par with the theory of classes and the theory of propositions. This paper presents a systematic analysis of symbolism in a simple and general form, not restricted to the use of any particular symbols for particular purposes. It is shown that any symbolic expression can be broken up into a number of "irreducible" expressions, such as $a+b, x=y, p \supset q$, and 
the like. The chief operation in the manipulations of symbols is that of replacing one of the variables in such an irreducible expression by some already constructed expression $E$, thus giving a more complex expression in which $E$ is a component. Using this operation, we can define the relation which makes one expression $A$ a case of a second expression $B$, and the converse relation in which $B$ is a form of $A$. These two relations are of great importance in the application of the theory of symbolism to logic. (Received March 9,1934.)

\section{Dr. Deane Montgomery (National Research Fellow):} A metrical property of point set transformations.

The following theorem is proved: If $E$ is a conditionally compact arc-wise connected metric space and if $T$ is any continuous one-one transformation of $E$ into itself, then there are two distinct points of $E$ whose distance remains invariant under $T$. This theorem applies in particular to open or closed spheres in $n$-dimensional space and to spheres in function space. (Received February 24, 1934.)

\section{Dr. G. T. Whyburn: Non-alternating transformations.}

If $A$ and $B$ are compact and metric, a continuous transformation $T(A)=B$ is said to be non-alternating if for no two points $x$ and $y$ of $B$ does the set $T^{-1}(x)$ separate two points of $T^{-1}(y)$ in $A$. A study is made of such transformations along with a similar investigation of those transformations in which the sets $T^{-1}(x)$ are all connected, the latter being equivalent to upper semicontinuous decompositions of $A$ into continua. Among other things it is shown that if $A$ is locally connected, each true cyclic element of $B$ is the image under some non-alternating transformation of a cyclic element in $A$. Various applications of the results to the cases of spheres, circles, cactoids, and boundary curves are obtained. (Received March 3, 1934.)

\section{Dr. John Williamson: The covariants of two quadratic} forms in $n$ variables.

Let $f$ and $g$ be two quadratic forms in the $n$ variables $x_{1}, x_{2}, \cdots, x_{n}$, with matrices $A$ and $R$ respectively. It is known that, if $\phi$ and $\gamma$ are the tangential forms of $f$ and $g$, the coefficients of the powers of $\lambda$ in the point form of the pencil $\phi+\lambda \gamma$ are projective covariants of $f$ and $g$ and, together with their jacobian $J$, form a complete system of covariants. We consider another set of $n$ quadratic covariants, namely those whose matrices are $R, A, A S A$, $A(S A)^{2}, \cdots, A(S A)^{n-2}$, where $S$ is the matrix adjugate to $R$. By means of this system we are able to show how the composition of certain of the irreducible concomitants of $f$ and of $g$ depends on the elementary divisors of $A+\lambda R$. In particular we show that, if $R$ is non-singular and the elementary divisors of $A+\lambda R$ are $\left(\lambda+\lambda_{i}\right)^{e_{i}}, i=1,2, \cdots, t ; e_{1}+e_{2}+\cdots+e_{t}=n, \lambda_{i} \neq \lambda_{j}$, if $i \neq j$, then the jacobian $J=I k_{1} e_{1} k_{2} e_{2} \cdots k_{t} e_{t}$, where $I$ is an invariant and $k_{1}, k_{2}, \cdots, k_{t}$ are $t$ linearly independent linear forms in $x_{1}, x_{2}, \cdots, x_{n}$. (Received March 2, 1934.) 


\section{Mrs. Grace M. Hopper and Professor Oystein Ore:} New types of irreducibility criteria.

Instead of the open Dumas polygon usually applied in the deduction of irreducibility criteria, a closed convex polygon is introduced, depending both on the size and the divisibility properties of the coefficients. For this polygon an approximate multiplication theorem holds and this may be used to deduce irreducibility criteria depending on the size of the coefficients. (Received March 7, 1934.)

\section{Mr. Saunders MacLane: On the representation of finite dual groups.}

Abstract dual groups, as discussed by Dedekind, F. Klein, and G. Birkhoff, include many common systems involving two operations, such as the operations of least common multiples and greatest common divisor or the operations (for classes) of meet and join. In particular, a dual group is of $i d e a l$ type when these operations satisfy, beside the usual axioms, both possible distributive laws. The chief result of the paper is the determination of all possible finite dual groups of this sort. This is done by first constructing a normal pointset representation of the group and then setting up a basis for this representation. The elements of the group can be uniquely represented in terms of this basis, and thus we obtain a finite analogue to some of the decomposition theorems of ideal theory. The use of a basis also gives a necessary and sufficient condition for the isomorphism of two groups and an analysis of the free groups on $n$ elements. Finally, certain relations of inclusion in the basis can be defined. For any preassigned basis with given inclusion relations there must exist a corresponding group. (Received March 9, 1934.)

\section{Dr. Ralph Hull (National Research Fellow): A canoni- cal generation of rational cyclic algebras of odd prime degree.}

Every rational cyclic algebra $A$ of odd prime degree $n$, that is, every cyclic algebra over the field $R$ of all rational numbers of order $n^{2}$ over that field, is shown to have a cyclic generation $A=\left(\sigma, Z_{p}, S\right)$ where $\sigma=1$ if $A$ is a total metric algebra, $\sigma=q_{1}, \cdots, q_{r}$ is a product of $r(\geqq 2)$ distinct primes $q_{i}$ if $A$ is a division algebra. The field $Z_{p}$ is cyclic over $R$ of degree $n$ over $R$ with a prime conductor $p \equiv 1(\bmod n)$, that is, $Z_{p}$ is the class field for the division of rational numbers prime to $p$ into $n$ classes consisting of the class of $n$-ic residues modulo $p$ and $n-1$ classes of $n$-ic non-residues modulo $p$. Each prime $q_{i}$ is distinct from $p$ and is an $n$-ic non-residue modulo $p$ while the $q_{i}$ are distributed in the $n-1$ classes of $n$-ic non-residues modulo $p$ in such a way that $\sigma$ is itself an $n$-ic residue modulo $p$. Finally, $S$ is a generating automorphism of the cyclic group of automorphisms of $Z_{p}$. The integer $\sigma$ is uniquely determined by $A$, the primes $q_{i}$ being precisely those primes for which the $A$-invariants $\nu_{q_{i}}$ (see Hasse, Transactions of this Society, January, 1932) are different from zero. For a given $\sigma$ there exist infinitely many primes $p$ with the properties described. (Received February 12,1934.) 
129. Dr. D. H. Lehmer: An extended theory of Bernoulli and Euler numbers.

It is customary to associate with the numbers $B_{n}, E_{n}$, of Bernoulli and Euler, two other sequences of numbers $G_{n}, R_{n}$ which are in fact the coefficients of the Maclaurin expansion of the tangent and cosecant. It is the purpose of this paper to discuss the properties of a set of $m^{2}$ sequences of rational numbers, which coincide for $m=2$ with the 4 already mentioned, and which are the coefficients of the Maclaurin expansions of the $m$ reciprocals and the $m(m-1)$ ratios of the $m$ Olivier functions of order $m$. The $k$ th Olivier function of order $m$ is a power series whose terms are obtained from the series for $e^{x}$ by selecting every $m$ th term beginning with the $k$ th term $(0 \leqq k<m)$. Most of the theory already developed for $B_{n}, E_{n}, R_{n}$, and $G_{n}$ can be extended to the general case of $m^{2}$ sequences. We have, for instance, generalizations of the fundamental recurrence formulas, the divisibility properties, periodicity with respect to a modulus, the von Staudt-Clausen theorem, etc. In order to express the terms of these sequences by infinite series, it is necessary to generalize the ordinary Dirichlet series to a sum which extends over the roots of the Olivier functions. Except for $n=2,4$, and 6 these roots are not distributed uniformly. (Received March 9, 1934.)

130. Dr. Selby Robinson: Pseudo-transitivity in finite and infinite groups.

A group is pseudo- $k$-fold transitive if it has elements that transform any unordered set of $k$ points into any other, and quasi- $k$-fold transitive if it has this property for every $r \leqq k$. It is shown in this paper that a quasi- $k$-fold transitive group $G$, of sufficiently high finite degree, is $k-2$ times transitive. Except when $5 \leqq k \leqq 8, G$ is $k-1$ times transitive; while if $k \leqq 10$ and composite, $G$ is $k$ times transitive. There are a number of theorems in the paper that apply to both finite and infinite groups. For example: a necessary and sufficient condition that a quasi-4-fold transitive group be doubly transitive is that it have a transformation which leaves a set of three points invariant but not pointwise invariant. (Received March 7, 1934.)

\section{Professor A. A. Albert: A solution of the principal prob- lem in the theory of Riemann matrices.}

The principal problem in the theory of Riemann matrices is that of determining the multiplication algebra $D$ of any pure Riemann matrix. Algebra $D$ is a normal division algebra over its centrum $K$ which is either a total real field or a pure imaginary quadratic extension of a total real field. The author solved the problem in the first case in an earlier paper. He now proves that in the second case the algebra $D$ is a certain type of cyclic algebra of degree $n$ over $K$, and that conversely there exist division algebras $D$ of the given type for any $n$ and pure Riemann matrices with $D$ as multiplication algebra. (Received March 7, 1934.) 


\section{Professor A. A. Albert: A matrix proof of the Poincaré theorem on impure Riemann matrices.}

The best proof of the Poincaré theorem on impure Riemann matrices in the literature is probably the very complicated projective geometric proof of G. Scorza (Palermo Rendiconti, 1916). The purpose of the present paper is to provide a purely algebraic proof of this important theorem as well as of the theory of the reduction of an impure Riemann matrix to pure components. The proofs given are far more elegant than any in the literature and provide the first purely algebraic treatment of the subject of impure Riemann matrices. (Received March 7, 1934.)

133. Professor A. A. Albert: Cyclic fields of degree $p^{n}$ over $F$ of characteristic $p$.

E. Artin and O. Schreier have given a determination of all cyclic fields of degree $p$ and $p^{2}$ over a field $F$ of characteristic $p$. In the present paper the author solves the corresponding problem in the case of degree $p^{n}$, the general case. (Received March 7, 1934.)

\section{Professor P. A. Caris: Integral solutions of $P^{4}+4 Q^{4}$} $=R^{4}+4 S^{4}$.

Numerical solutions of the diophantine equation $P^{4}+k Q^{4}=R^{4}+k S^{4}$, where $k$ is given, have been found by several writers for various values of $k$. But the only case in which parametric solutions have been obtained hitherto seems to be the case $k=1$ for which Euler found a two-parameter solution involving polynomials of the seventh degree. In the present paper a parametric solution is found for the case $k=4$. The method is different from that of Euler. The result is $\left(a^{3}-4 a^{2} b-2 a b^{2}-4 b^{3}\right)^{4}+4\left(a^{3}+a^{2} b+4 a b^{2}-2 b^{3}\right)^{4}=\left(a^{3}+4 a^{2} b-2 a b^{2}\right.$ $\left.+4 b^{3}\right)^{4}+4\left(a^{3}-a^{2} b+4 a b^{2}+2 b^{3}\right)^{4}$. (Received March 2, 1934.)

135. Dr. J. L. Dorroh: Concerning the direct product of algebras.

This paper gives the conditions under which two algebras which do not possess a finite basis have a direct product. (Received March 6, 1934.)

\section{Mr. Garrett Birkhoff: On the lattice theory of ideals.}

A uniqueness theorem on the direct decomposition of finite lattices is proved, together with a necessary and sufficient condition for the existence of such a decomposition in the case of $B$-lattices. On this basis the combinatorial relations of "einartig" ideals in a commutative ring with unity are discussed, and a criterion that the ideals should combine like point sets is derived. (Received February 7, 1934.)

\section{Professor Edward Fleisher: On Euler squares.}

In the Annals of Mathematics, vol. 23, No. 3, March, 1922, H. F. MacNeish gave a proof of the impossibility of the construction of Euler squares of order 
$n$, degree 2 , index $(n, 2)$ (Graeco-Latin squares) when $n \equiv 2(\bmod 4)$ for the case in which the square, interpreted as a set of 2-circuits, lies on a manifold or on a set of manifolds in which each vertex is taken with the same multiplicity. All known Graeco-Latin squares have this property but no proof that the condition is necessary appears in the literature. The present author considers Euler squares of index $(n, 2)$ which are formed by operating on either the first row or the first column of dyads by means of a regular group of substitutions. This is true of all known squares. He shows that a square of index $(r s, \nu)$ formed by the composition of squares of indices $(r, \nu)$ and $(s, \nu)$ is based on a group which is the direct product of the groups of the component squares. He proves that no Euler square of order $n$, degree 2, based on a group, can be formed when $n \equiv 2(\bmod 4)$. He also gives a linear-graph equivalent of the theorem that no Euler squares of index $(n, 3)$ can be constructed when $n \equiv 3$ $(\bmod 9)$. (Received February 12, 1934.)

\section{Dr. R. D. James (National Research Fellow): On the expansion coefficients of the functions $u / s n u$ and $u^{2} / s n^{2} u$.}

The values of certain coefficients in the expansion of $u / \mathrm{sn} u$ and $u^{2} / \mathrm{sn}^{2} u$ were stated without proof by Hermite (Oeuvres, vol. 3, pp. 236-237), and no proof seems to have been published. In this note a proof is given following the method of Gruder (Wiener Sitzungsberichte, IIa, vol. 126 (1917)). The proof depends on several recursion formulas for the Bernoulli numbers. (Received February 28, 1934.)

\section{Mr. Rufus Oldenburger: Transposition of indices in multiple-labeled determinants.}

An ordinary determinant with more than one index associated with its rows or columns is called a multiple-labeled determinant. It is shown in this paper how these determinants arise under non-singular linear transformations on multilinear forms, where they possess relative invariant properties, and if sufficiently specialized factor into determinants of lower orders. The effect of transpositions of indices on the value of a multiple-labeled determinant, its minors, and cofactors, is then determined. The latter problem resolves itself into a study of the evenness or oddness of the substitutions necessary to bring a multiple-labeled array of numbers of the type $11,12, \cdots, 1 n, 21, \cdots$, $2 n, \cdots, m 1, \cdots, m n$ into the array $11,21, \cdots, m 1,12, \cdots, m 2, \cdots, 1 n$, $\cdots, m n$. Finally, it is shown how the matrices associated with multiplelabeled determinants are related to matrices used by Dirac and Weyl. (Received March 3, 1934.)

\section{Mr. Rufus Oldenburger: Factorization of hyperspace} matrices into non-singular matrices.

Necessary and sufficient conditions for a matrix $A=\left(a_{i_{1}} \cdots i_{p}\right)$ to factor in the form $\left(a_{\alpha i_{p}}{ }^{(1)} \cdots a_{\alpha i_{p}}{ }^{(p)}\right)$, where $\left(a_{\alpha i_{1}}{ }^{(1)}\right), \cdots,\left(a_{a i_{p}}{ }^{(p)}\right)$ are matrices nonsingular on the index $\alpha$ (rows), are given by the author in another paper. In the present paper necessary and sufficient conditions are given for the factorization 
of the matrices $A=\left(a_{i j_{1}} \cdots j_{p}\right)$ and $A=\left(a_{i j k_{1}} \cdots k_{p}\right)$. The problem resolves itself into a problem in the equivalence of $n$-tuples of matrices. (Received March 3, 1934.)

141. Dr. M. R. Hestenes (National Research Fellow): Sufficient conditions for the problem of Bolza in the calculus of variations. II.

Until recently sufficient conditions for a minimum in the problem of Bolza have been given only for arcs which are not only normal relative to the end conditions but also normal on every subinterval. In a first paper (abstract 39-7-213) the author has shown that by a suitable modification of the condition of Mayer a set of sufficient conditions is obtained involving the assumption of normality relative to the end conditions but not the assumption of normality on the subintervals. In the present paper it is shown that the assumption of normality relative to the end conditions also is not needed. We merely need the assumption that there exists a set of multipliers of the form $\lambda_{0}=1, \lambda_{\beta}(x)$ with which the arc under consideration satisfies the usual sufficient conditions except possibly for a suitable modification of the condition of Mayer. In the normal case these sufficient conditions are identical with the known ones. (Received February 23, 1934.)

142. Mr. F. J. Murray: Linear transformations between distinct Hilbert spaces.

The usual theory of linear transformations in Hilbert space is first generalized to the theory of linear transformations between Hilbert spaces. For $T$ continuous and linear we obtain a method for the computational determination of the general solution of the equation $T f=g$. For $T$ closed and linear, we determine the set of all closed linear manifolds $\mathfrak{M}$ such that if $f_{\epsilon} \mathfrak{D}$, then $f=f_{1}+f_{2}$, where $f_{1} \in \mathfrak{M} \cdot \mathfrak{D}, f_{\in} \mathfrak{D} \cdot \mathfrak{h} \ominus \mathfrak{M}$ and $T f_{1}$ is orthogonal to $T f_{2}$. The theory of such manifolds for a closed linear transformation is quite analogous to the theory of manifolds which reduce a self-adjoint transformation. By their use we develop methods which compensate, in the study of possible inverses of a closed linear transformation, for the lack of compactness of Hilbert space. (Recived March 5,1934 .)

143. Dr. A. H. Smith: Summability of the derived conjugate Fourier series. Preliminary report.

B. N. Prasad (Journal de Mathématiques Pures et Appliquées, (9), vol. 11 (1932), p. 178) has proved a theorem concerning the Cesàro summability of the conjugate Fourier series. Using the Bosanquet-Linfoot method of summation (Journal of the London Mathematical Society, vol. 6 (1931), pp. 117-126), we extend in a similar manner the results of an earlier paper (this Bulletin, abstract 39-9-262) concerning the summability of the $r$ th, $r \geqq 0$, derived conjugate Fourier series of a function $f(x) \subset \&$. A more general result than Prasad's is obtained as a special case, namely when $r=0$, since the BosanquetLinfoot method of summation is weaker than that of Cesàro. (Received March 7, 1934.) 
144. Dr. Deane Montgomery (National Research Fellow): Properties of functions of two variables.

If $p$ is any property of functions of one variable and if $f(x, y)$ is any function of two variables, it is an interesting problem to determine the nature of the set of $\bar{x}$ 's for which $f(\bar{x}, y)$ has the property $p$. This problem is completely solved in this paper when $f(x, y)$ is in the Baire classification and when $p$ is any one of several properties among which are Riemann integrability, differentiability, and the property of having bounded variation. (Received February 24, 1934.)

145. Dr. H. W. Raudenbush: Hypertranscendental extensions of partial differential fields.

Differential fields, as defined by R. Baer, have a role in the abstract or formal theory of ordinary algebraic differential equations analogous to the role of field in abstract algebra. With an abstract theory of partial differential equations in view, the analogous concept of partial differential field is defined. The analogues to Steinitz' theorems on transcendental extensions of fields, obtained previously for differential fields, are carried over to partial differential fields. (Received March 5, 1934.)

\section{Dr. J. L. Doob (National Research Fellow) : Probability distributions and statistics.}

Let $\mathrm{x}$ be a chance variable which can take on any real value and let $F(x)$ be the probability that $\mathrm{x}<x$. For $n$ repeated trials a new set-up is needed consisting of $n$-dimensional euclidean space with a metric determined by $F(x)$. It is shown that there is a space of infinitely many dimensions which serves for an experiment with an arbitrary number of trials. This space is the space whose points are infinite sequences: $\cdots x_{-1}, x_{0}, x_{1}, \cdots$ where $x_{j}$ is any real number, with a probability function (i.e., a completely additive non-negative set function with value 1 on the whole space) defined on it. By the use of the ergodic theorem of Birkhoff a generalization of the Law of Large Numbers is obtained: namely, that a necessary and sufficient condition that in a repeated trial $\lim _{n \rightarrow \infty} \sum_{1}^{n}\left(x_{j} / n\right)=c$ (where $c$ is some constant) with probability 1 , is that the expectation of the chance variable exists, and if this is so, $c$ is this expectation. (This result was announced without proof by Khintchine.) These results are applied to prove rigorously certain well known theorems of R. A. Fisher used in statistics, concerning the principle of maximum likelihood. (Received March 9, 1934.)

147. Professor J. F. Ritt: On the order of a system of algebraic differential equations.

This paper presents rigorous results relative to the number of arbitrary constants in the solution of a finite system of algebraic differential equations. The orders to which the unknowns appear in the equations being given, upper bounds are secured for the number of constants in the solutions of the irreducible systems into which the given system decomposes. (Received March 10, 1934.) 


\section{Dr. I. J. Schoenberg: On asymptotic distributions of arithmetical functions.}

In 1928 the author proved (Mathematische Zeitschrift, vol. 28) that the sequence $\phi(n) / n(n=1,2,3, \cdots ; \phi(n)$ is the Euler function) has a continuous asymptotic distribution over the interval $(0,1)$. Using the author's method, H. Davenport proved recently (Sitzungsberichte der Preussischen Akademie, 1933 ) the following general theorem: Let the arithmetical function $f(n)$ satisfy the following conditions: (1) $0<f(n) \leqq 1$; (2) $f(m n)=f(m) f(n)$ if $(m, n)=1$; (3) with positive constants $C, c$ we have $0 \leqq f\left(p^{e-1}\right)-f\left(p^{e}\right) \leqq C p^{-c e}$ for all primes $p$ and $e \geqq 1$; (4) the numbers $\log f(p)$ are linearly independent for all sufficiently large primes $p$. Then the sequence $f(n),(n=1,2,3, \cdots)$, admits a continuous asymptotic distribution over $(0,1)$. In the present note, by essentially different methods, the following theorem is derived. If $f(n)$ satisfies the conditions $\left(1^{\prime}\right) f(n)>0,(2)$ as above, $\left(3^{\prime}\right)$ the series $\sum_{p}|\log f(p)| / p$ converges, then $f(n)$ has already an asymptotic distribution over $(0,+\infty)$ which however need not be continuous. This distribution is certainly continuous if also (4) holds. It may be emphasized that this last theorem makes no assumptions whatever about the values $f\left(p^{e}\right)$ for $e>1$, except that they be positive. (Received March 9, 1934.)

149. Professor Oystein Ore: Remarks on polynomials taking prime values.

Pólya, A. Brauer, H. Dorwart, and the author have investigated the irreducibility of polynomials taking a fixed prime value a certain number of times. In the present paper the results are generalized to polynomials taking any prime values. The exact upper limit for the number of prime values which a reducible polynomial may take is obtained. Certain criteria of a different type for irreducibility are also derived. (Received February 28, 1934.)

\section{Dr. G. A. Hedlund (National Research Fellow): $A$ metrically transitive system.}

It has been known for some time that there exist transitive geodesics on closed orientable surfaces of genus greater than one and of everywhere negative curvature. The question as to whether regional transitivity implies metrical transitivity has not been answered. But it can be shown that in the case of a certain set of surfaces which are closed, orientable, of genus greater than one, and of constant negative curvature, the system of geodesics has the property of metrical transitivity. The result is described in detail in a note $O n$ the metrical transitivity of the geodesics on a surface of constant negative curvature, in the February issue (1934) of the Proceedings of the National Academy of Sciences. (Received February 20, 1934.)

151. Dr. A. B. Brown: Note on the form of a first-order partial differential equation.

A simple direct proof is given of the possibility of replacing a first-order partial differential equation by one which does not involve the dependent vari- 
able explicitly. The usual proof by use of the "complete integral" uses an elaborate machinery and requires a considerable amount of detailed work to be made complete. (Received February 5, 1934.)

\section{Professor O. J. Farrell: On approximation to an analytic} function by polynomials.

The chief results obtained are as follows: (1) if the function $f(z)$ is analytic and bounded in a finite simply connected region $R$ whose boundary divides the $z$-plane into just two regions, there exist polynomials $p_{n}(z), n=1,2, \cdots$, that converge to $f(z)$ in $R$ as $n \rightarrow \infty$ in such a way that the limit (as $n \rightarrow \infty$ ) of the least upper bound of $\left|p_{n}(z)\right|$ for $z$ in $R$ does not exceed the least upper bound of $|f(z)|$ for $z$ in $R$; (2) if the function $f(z)$ is analytic in a finite simply connected region $R$ whose boundary divides the z-plane into just two regions and if the double integral over $R$ of the $p$ th power $(0<p)$ of $|f(z)|$ exists, then there exist polynomials $p_{n}(z), n=1,2, \cdots$, which converge to $f(z)$ in $R$ as $n \rightarrow \infty$ in such a way that the limit (as $n \rightarrow \infty$ ) of the double integral over $R$ of the $p$ th power of $\left|f(z)-p_{n}(z)\right|$ is zero. A comparison of the measures of convergence involved in these two theorems is made in the case of a particular example. It is shown that the first of these theorems cannot be extended to the most general finite simply connected region. (Received February 21, 1934.)

\section{Dr. E. K. Haviland: On the determinateness of the multi- dimensional momientum problem.}

Let $\phi_{i}, i=1,2$, be distribution functions, i.e., monotone absolutely additive set functions defined throughout the $x y$-plane, $S$, and possessing the total variation 1 there. Furthermore, suppose the momenta of $\phi_{1}$ and $\phi_{2}$ exist and are the same, so that $G_{p q}=\iint_{s} x^{p} y^{q} d_{x y} \phi_{i}(E)$ for $i=1,2$ and all non-negative values of the integers $p$ and $q$. Then a sufficient condition that $\phi_{1}=\phi_{2}$ is that the $2 m$ th root of $\sum_{\nu=0}^{2 m}\left(\begin{array}{c}2 m \\ \nu\end{array}\right)\left|G_{2 m-\nu, \nu}\right|=o(m)$. Moreover, the condition is almost necessary in that there are examples showing that $\phi_{1}$ need not equal $\phi_{2}$ if $o(m)$ is replaced by $o\left(m^{1+\epsilon}\right)$. This theorem may easily be extended to $n$-dimensional spaces, $n>2$, and may be applied to a deduction of the multi-dimensional Gaussian distribution. (Received March 9, 1934.)

154. Mr. F. J. Murray: Partial differential operators as linear transformations between Hilbert spaces.

We define a function space $\mathfrak{W}$, in which the elements are functions of $(x, y)$ on a bounded region $S$ and the scalar product is $(u, v)=\iint_{S}\left(u_{x x} \bar{v}_{x x}+u_{x y} \bar{v}_{x y}\right.$ $\left.+u_{y x} \bar{y}_{y x}+u_{y y} \bar{v}_{y y}+u_{x} \bar{v}_{x}+u_{y} \bar{v}_{y}+u \bar{v}\right) d S$. We show that $\mathfrak{W}$ is a Hilbert space. A linear partial differential expression $L(u)$ of the second order with bounded measurable coefficients represents a bounded transformations from $\mathfrak{W}$ to $\Omega_{2}$, the Hilbert space of scalar product $(u, v)=\iint_{S} u \bar{v} d S$. We then further limit $B$, the boundary of $S$, to be a rectifiable curve with certain other restrictions. Let $\beta(u)$ be a linear combination of the boundary values of $u, u_{x}$, and $u_{y}, u \in \mathfrak{W}$, with coefficients which are bounded measurable functions of the arc-length $s$. Then $w=\beta(u)$ represents a bounded transformation from $\mathfrak{W}$ to $\mathbb{S}$, the space 
of functions defined on the boundary, of scalar product $(u, v)=\int_{\beta} u \bar{v} d s$. We then give methods for the computation of all $u \in \mathfrak{W}$, such that $L(u)=v$ and $\beta(u)=w$ for a given $v \epsilon \mathfrak{R}_{2}$ and $w \epsilon$ (5). (Received March 7, 1934.)

\section{Professor John von Neumann: Almost periodic functions in a group.}

In this paper the notion of almost periodic functions due to $\mathrm{H}$. Bohr is generalized to arbitrary groups $G$. By varying the form of the definition of these functions due to $\mathrm{S}$. Bochner, the notion becomes entirely independent of continuity, or of any sort of topology in $G$. The main difficulty is to define an equivalent of $\mathrm{H}$. Bohr's "integral mean." This is done by an entirely new process of defining the "mean" (which, for instance, in the Bohr case, applies even to non-measurable functions). After this has been done, the operational method of Weyl can be applied, and leads to the "fundamental" and "approximation" theorems, corresponding to those of Bohr. The theory which is thus obtained permits generalization of the Frobenius-Schur theory of representations, beyond its extension by Weyl-Peter, to all bounded representations of any group $G$. The familiar theorems of completeness hold. The continuity of all expansion terms of continuous almost periodic functions is established (if $G$ is topological), and various special $G$ 's are discussed. The existence of a "maximal" set of "characteristic" and of almost periodic functions (in a certain precise sense) is proved for locally compact Abelian groups by the use of Haar's generalized integral, and various theorems of the author on operators. (Received February 23, 1934.)

\section{Professor J. F. Ritt: Algebraic difference equations.}

It was known in the second half of the eighteenth century that non-linear algebraic difference equations, even if algebraically irreducible, might have more than one solution depending on an arbitrary periodic function. This paper gives a bound for the number of such solutions. (Received March 10, 1934.)

157. Professors George Rutledge and R. D. Douglass: $A$ hypergeometric parametric function defining the range of de la Vallée Poussin summation.

The functions $Q(x, z)=F(x,-x, 1, z)$ and $-\partial Q / \partial z$ are integral functions of $x$ defined by Stirling interpolation series with $z$ as parameter, $-1 \leqq z<1$. For $z=-1$, these functions are denoted by $Q(x), x M_{1}(x)$, respectively. They have positive integer values for positive integer values of $x$, and as $x$ becomes infinite, $x=n, Q(n) \cong 2^{1 / 2} M_{1}(n) \cong 2^{1 / 4} \cosh \left[\alpha n /(\pi n)^{1 / 2}\right]$, where $\alpha=2 \quad \sinh ^{-1} 1$ $=\log \left(3+2^{3 / 2}\right) .(V P) \sum_{0}^{\infty} u_{n}$ and $u_{0}+\left(V P^{\prime}\right) \sum_{1}^{\infty} u_{n}$ denote identical processes of summation. (See The inverse matrix for de la Vallée Poussin summation, Journal of Mathematics and Physics of the Massachusetts Institute of Technology, vol. 11 (1932), pp. 73-82.) It is convenient to consider $\left(V P^{\prime}\right) \sum_{1}^{\infty} u_{n}$. The $\left(V P^{\prime}\right)$ transforms of the divergent alternating series $\sum_{1}^{\infty}(-1)^{n-1} Q(n)$, $\sum_{1}^{\infty}(-1)^{n-1} 2 M_{1}(n) / n$ are, respectively, $\sum_{1}^{\infty}(-1)^{n-1}, \sum_{1}^{\infty}(-1)^{n-1} 1 / n$. The function $Q(n)$ serves the same purpose for $\left(V P^{\prime}\right)$ summation (and hence also 
for (VP) summation) as the function $n^{k}$ for Cesàro $C_{k}$ summation. A necessary condition that $\sum_{1}^{\infty} u_{n}$ shall be $\left(V P^{\prime}\right)$ summable is that $u_{n}=o\{Q(n)\} . Q(n)=2,8$, $38,192,1002, \cdots$, and $M_{1}(n)=1,5,25,129,681, \cdots$, satisfy simple recursion formulas. (Received February 19, 1934.)

158. Miss Norma Stelford and Professor H. A. Simmons: Classes of maximum numbers associated with certain symmetric equations in $n$ reciprocals.

In a previous paper dealing with this subject (Transactions of this Society, vol. 34 , no. 4 , p. 876$)$ the authors considered certain symmetric equations with right members of the form $b / a, a \equiv(c+1) b-1$, and thus equal to a sum of two unit fractions. In the present paper a much milder restriction is made on the nature of $b / a$, and analogs of the theorems relative to maximum numbers in the article referred to above are still obtained. Thus the main results of that article are generalized considerably. (Received February 17, 1934.)

159. Professor H. A. Simmons: The Kellogg solution of a cyclo-symmetric equation; questions concerning extreme numbers relative to this solution.

Let $C_{t}(1 / x)$ be the following cyclo-symmetric function of the $n$ reciprocals $\left(1 / x_{i}\right), \quad(i=1,2, \cdots, \quad n), \quad n>t: \quad C_{t}(1 / x)=\left(1 / x_{1} \cdots x_{t}\right)+\left(1 / x_{2} \cdots x_{t+1}\right)$ $+\cdots+\left(1 / x_{n} x_{1} \cdots x_{t-1}\right)$. In this paper the author obtains the Kellogg solution (Transactions of this Society, vol. 34 , no. 4, p. 884) of the equation (1), $C_{r}(1 / x)+\lambda_{r+1} C_{r+1}(1 / x)+\cdots+\lambda_{s} C_{s}(1 / x)=b /[(c+1) b-1]$, where the $\lambda$ 's, $b, c$ are integers $\geqq 0$. Then certain maximum numbers and certain minimum numbers relative to special cases of equation (1) are identified. Certain problems which remain unsolved are proposed for (1) and other more general equations somewhat like (1). (Received February 17, 1934.)

160. Mr. F. C. Smith: On the asymptotic developments of analytic functions.

In a series of papers published during the years 1900-1908, E. W. Barnes obtained the asymptotic developments of a large number of function types by means of highly specialized methods. Systematic methods of obtaining such asymptotic expansions have been developed in more recent years by W. B. Ford and C. V. Newsom. In this paper, the general theory is used in considering the asymptotic behavior of the following function types: (1) $f(z)=\sum_{n=0}^{\infty} z^{n} /(n+\theta)^{\beta} ;$ (2) $f(z)=\sum_{n=0}^{\infty} h(n) z^{n} / \Gamma(n+p)$. (Received February 24, 1934.)

161. Dr. J. I. Vass: A class of boundary problems of highly irregular type.

J. W. Hopkins (Transactions of this Society, vol. 20 (1919), p. 245) and L. E. Ward (Annals of Mathematics, vol. 26 (1925), p. 21; also, Transactions of this Society, vol. 29 (1927), p. 716) have considered the problem of the expansion of a function $f(x)$ in a series of solutions of ordinary linear differential 
systems, in which the differential equation, $d^{n} u / d x^{n}+\rho^{n} u=0, n \geqq 3$, ( $\rho$ a complex parameter) is associated with boundary conditions of highly irregular type. The present paper takes up the expansion problem for certain highly irregular systems in which the order is reduced, $n=2$, and the element of symmetry, present in the solutions of the above equation, plays no important role. Two distinct systems involving the equation $d^{2} u / d x^{2}-2 \rho \cos c d u / d x+\rho^{2} u=0$, $c=(p / q) \pi, 0<2 p<q$, are discussed with the purpose of exhibiting the true nature of such expansion problems. For the systems considered, sufficient conditions upon $f(x)$ are developed under which the expansion for the function converges uniformly or is summable by an application of the Riesz typical means. Comparisons are made with the work of Hopkins and Ward and also with the corresponding situations for systems which are regular or mildly irregular. (Received February 28, 1934.)

162. Professor C. C. MacDuffee and Mr. E. D. Jenkins: $A$ substitute for the Euclid algorithm in algebraic fields.

A method free from tentative steps is outlined for computing the greatest common divisors (provided they exist) of two or more integral numbers of any quadratic field. The method involves the use of matrices with rational integral elements. Except for one step involving the solution of a diophantine equation, the method is applicable to algebraic fields of higher order, and a tentative method will often give at least one greatest common divisor in these cases. To show the ease and rapidity of calculation by this method, examples are given in $F\left[(53)^{1 / 2}\right]$ and in $F(\theta)$ where $\theta^{3}+6 \theta+8=0$, the latter being a cubic field of class number 3. (Received March 1, 1934.)

\section{Mr. Rufus Oldenburger: Canonical triples of bilinear} forms.

In this paper are given the canonical forms to which triples of bilinear forms can be reduced under non-singular linear transformations in the complex field where a pair of bilinear forms in each triple has associated with it distinct characteristic roots. Complete systems of invariants for triples are obtained from the canonical forms. (Received March 2, 1934.)

164. Mr. Rufus Oldenburger: Factorization of hyperspace matrices into a product of two-way matrices one of which is singular.

In another paper the author has given necessary and sufficient conditions for a matrix to be of the form $\left(a_{\alpha i_{1}}^{(1)} \cdots a_{\alpha i_{n}}^{(n)}\right)$, where the matrices $\left(a_{\alpha i_{1}}^{(1)}, \cdots, a_{\alpha i_{n}}^{(n)}\right)$ are non-singular on $\alpha$ (rows are linearly independent). In the present paper necessary and sufficient conditions are obtained for a matrix to be of the above form where $\left(a_{\alpha i_{1}}^{(1)}\right)$ is singular on $\alpha$, and $\left(a_{\alpha i_{2}}^{(2)}\right), \cdots,\left(a_{\alpha i_{n}}^{(n)}\right)$ are non-singular on $\alpha$. In solving the problem it is necessary to obtain necessary and sufficient conditions for a set of matrices to be simultaneously equivalent under non-singular linear transformations to a set of diagonal matrices. These conditions are given in this paper. (Received March 2, 1934.) 
165. Mr. Rufus Oldenburger: Non-singular multilinear forms.

A multilinear form equivalent to $x_{\alpha} y_{\alpha} \cdots z_{\alpha} w_{\alpha}$ is called a non-singular form. Let $\delta=\left(\delta_{i j} \ldots k l\right)$, where $\delta_{i j} \ldots k l=1$ for $i=j=\cdots=k=l=1, \cdots, n$, $\delta_{i j} \ldots k l=0$ for $(i, j, \cdots, k, l) \neq(1,1, \cdots, 1,1), \cdots,(n, n, \cdots, n, n)$. The matrix $\delta$ and all matrices equivalent to $\delta$ under non-singular linear transformations are called non-singular matrices. Necessary and sufficient conditions for the non-singularity of a trilinear form have been given in another paper by the author. In the present paper such conditions are given for the non-singularity of an $r$-linear form, where $r \geqq 4$. The determination of these conditions depends largely on the determination of necessary and sufficient conditions for an $m$-tuple of multilinear forms, where one of the forms is non-singular, to be equivalent to a set of multilinear forms with diagonal matrices. (Diagonal matrices are matrices whose only non-vanishing elements are elements determined by setting all indices equal.) It is shown that a matrix $\left(g_{i} \ldots l\right)$ is nonsingular if and only if it can be written in the form $\left(a_{\alpha i} \cdots d_{\alpha l}\right)$, where $\left(a_{\alpha i}\right), \cdots,\left(d_{\alpha l}\right)$ are non-singular two-way matrices. Finally, necessary and sufficient conditions for a matrix to be of the form $\left(a_{\alpha i} \cdots d_{\alpha l}\right)$, where $\left(a_{\alpha i}\right), \cdots,\left(d_{\alpha l}\right)$ are only assumed to be non-singular on $\alpha$ (rows), are written down. (Received March 2, 1934.)

166. Mr. Rufus Oldenburger: Solution of a class of simultaneous matrix and integral equations.

It is proved in this paper that the condition $C E^{\prime}=C^{\prime} E$ is necessary and sufficient for the existence of a non-singular matrix solution $Y$ of the equations $C Y=C^{\prime}, Y E=E^{\prime}$, where $C, C^{\prime}, E, E^{\prime}$ are non-vanishing vectors. This property is generalized to denumerably infinite matrices and integral equations, and related to the problem of solving the equations $X A Y=A^{\prime}, C Y=C^{\prime}, X B=B^{\prime}$ for non-singular matrices $X, Y$, where $A, A^{\prime}$ are non-singular matrices, and $C, C^{\prime}, B, B^{\prime}$ are vectors. Limiting cases where some of the vectors vanish are also considered. (Received March 2, 1934.)

\section{Mr. Rufus Oldenburger: Space ranks of the multiple composite of hyperspace matrices.}

The composite of two matrices $\left(a_{\rho i_{1} \cdots i_{m}}\right),\left(b_{\rho_{1} \cdots j_{n}}\right)$ may be formed by combining indices in essentially two ways: by writing $\left(a_{\rho i_{1}} \cdots i_{m} b_{\rho i_{1}} \cdots j_{m}\right)$ where $\rho$ is summed, and $\left(a_{\rho i_{1}} \cdots i_{m} b_{\rho j_{1}} \cdots i_{n}\right)$ where $\rho$ is not summed. Where the composite of two or more matrices is made by summing on at least one combined index and not summing on at least one other combined index we have the multiple composite of these matrices. In this paper certain relations are derived between the space ranks of the multiple composite of a set of matrices, obtained by combining indices in any way, and the space ranks of these matrices. (Received March 2, 1934.)

\section{Professor L. E. Dickson : New universal Waring theorems.}

The content of this paper is sufficiently indicated by the title. (Received March 5, 1934.) 
169. Dr. E. F. Beckenbach: A characteristic property of surfaces of negative curvature.

It has been pointed out (Transactions of this Society, vol. 35 (1933), p. 663) that if a surface $S$ of non-positive Gaussian curvature $K$ is given in conformal representation, $x_{j}=x_{j}(u, v)$ with $\sum x^{2}{ }_{j u}=\sum x^{2}{ }_{j v}=\lambda(u, v), \sum x_{j u} x_{j v}=0$, then $\lambda(u, v)$ is subharmonic (and even that a necessary and sufficient condition that $K \leqq 0$ on $S$ is that $\log \lambda$ be subharmonic). Examples are now given of particular conformal representations of surfaces with $K>0$ for which $\lambda(u, v)$ is subharmonic; nevertheless, the following converse of the above result is obtained: If for all conformal representations of a surface $S$ on $(u, v)$-domains, $\lambda(u, v)$ is subharmonic, then $K \leqq 0$ on $S$. As a result, it follows that the familiar inequalities of function theory, $a(r) \geqq \pi r^{2} \lambda\left(u_{0}, v_{0}\right), l(r) \geqq 2 \pi r \lambda\left(u_{0}, v_{0}\right)^{1 / 2}$, where $a(r)$ and $l(r)$ denote the area and length of boundary of the image of $\left(u-u_{0}\right)^{2}$ $+\left(v-v_{0}\right)^{2} \leqq r^{2}$ on $S$, are characteristic of surfaces with $K \leqq 0$. (Received March 5,1934 .)

170. Dr. R. D. James (National Research Fellow): The representation of integers as sums of values of cubic polynomials.

A cubic polynomial in $x$ is an integer for all integers $x \geqq 0$ if and only if it has the form $P(x)=a\left(x^{3}-x\right) / 6+b\left(x^{2}-x\right) / 2+c x+d$, where $a, b, c$, and $d$ are integers. Under the assumptions that $a, b$, and $c$ have no common factor, $a>0$, and $d=0$, it is proved in this paper that every sufficiently large integer is a sum of nine values of $P(x)$. The method of proof is that of Landau (Zum Waringschen Problem, Dritte Abhandlung, Mathematische Zeitschrift, 32 (1930), pp. 699-702). (Received March 5, 1934.)

171. Mr. G. C. Webber: Waring's problem for cubic functions.

Recent work by L. E. Dickson has thrown much light on the number of functional values necessary for the representation of an integer as a sum of positive integral values of a cubic function. The author considers certain sets of functions $f(x)=p\left(x^{3}-x\right) / 6+q\left(x^{2}-x\right) / 2+u x, p>0, q \neq 0, p, q$, and $u$ being integers. By a substitution $x=X+t, t$ an integer, $f(x)$ is transformed into $F(X)=p\left(X^{3}-X\right) / 6+g X+a$. It is proved that every integer exceeding $s_{0}$, say, is a sum of nine or ten values of $F(X)$ for values $\geqq|t|$ of $X$. This enables us to show that every integer exceeding a constant $s_{1}$ is a sum of nine or ten values of $f(x)$ for values $\geqq 0$ of $x$. These constants $s_{0}$ and $s_{1}$ are readily determined. A separate discussion for cubic functions without square term completes the investigation initiated by Dickson (Transactions of this Society, vol. 36, pp. 1-12). (Received March 5, 1934.)

172. Professor Dunham Jackson: The summation of series of orthogonal polynomials.

The summability of a development in series of orthogonal polynomials or orthogonal trigonometric sums by the first arithmetic mean is discussed by a method which involves a somewhat restrictive hypothesis as to the continuity of the function represented, but admits broad generality in the character of the 
weight function for which the orthogonal system is defined. (Received March 6, 1934.)

173. Mr. O. K. Sagen: The integers represented by positive sets of ternary quadratic non-classic forms.

This paper treats the problem of determining all integers represented by a set of positive ternary quadratic non-classic forms having given determinant. For given $a$ and $d$, conditions that there exist integers satisfying the equation $d+a r+b s=a b c$ are found. From these results it is shown that the set of forms having determinant $2 d$ represents all integers except those in certain arithmetical progressions. (Received March 6, 1934.)

174. Professor A. R. Crathorne: A reduction formula for the moments of a binomial distribution about the origin.

If $m_{i}$ is the $i$ th moment of a binomial distribution, $(p+q)^{n}$, about the origin, then $m_{n+1}=m_{1} m_{n}+p q d m_{n} / d p$. The formula is derived by algebraic manipulation from the well known general relation between semi-invariants and moments, together with the more special known relationships among moments and semi-invariants for the binomial distribution. (Received March 8, 1934.)

\section{Professor Wilhelm Maier: Concerning cubic theta func- tions.}

Following Riemann and Appell, the infinite series $\sum_{h=-\infty}^{+\infty} e^{2 \pi\left(x h+y h^{2} / 2+z h^{3} / 3\right)}$ $=\theta\left\{\begin{array}{l}x \\ y\end{array}\right\}$, which converges for $0<F(y)$ and $0=F(z)$, defines an analytic function of $x, y$, and $z$. To get the analytic continuation of that function for non-real values of $z$, a new expansion of $\theta\left\{\begin{array}{l}x \\ y\end{array}\right\}$ is given in terms of cyclindrical functions. (Received March 8, 1934.)

\section{Dr. E. S. Akeley: On a type of action integral in physics} and a certain transformation.

Let $L$ be an invariant function of a positive definite metric tensor $g_{i j}$ in four independent variables and its associated contracted Riemannian Christoffel tensor $R_{i j}$. Consider the invariant integral $I=\int L g^{1 / 2} d x^{(1)} \cdots d x^{(4)}$ and the associated Euler equations for the calculus of variations problem $\delta I=o\left(\delta g_{i j}\right)$. We obtain six independent fourth order equations for the ten $g_{i j}$. Furthermore, consider the transformation from $\left(g_{i j}, R_{i j}\right)$ to $\left(\lambda_{m}, p_{i m}\right)$ where $g_{i j}=\sum_{m} p_{i m} p_{j m}$ and $R_{i j}=\sum_{m} \lambda_{m} p_{i m} p_{j m} ; L$ becomes a symmetric function of the $\lambda_{m}$ and we obtain sixteen second order equations for the twenty $\left(\lambda_{m} p_{m}\right)$. If, furthermore, the coordinate system is canonicalized (See abstract 140-1-19) so that $\lambda_{m}=x_{(m)}^{(m)}$ we obtain sixteen independent second order equations for the sixteen functions $p_{i m}$, out of which four first order equations are deducible. If one considers the coordinate system canonicalized in the original integral, the new variation problem is of the form $\delta I=o\left(\delta p_{i m}\right)$ under an auxiliary condition which introduces a Lagrangian tensor. The equations for this problem are shown to be equivalent to the original equations. These results can be easily generalized for a non-definite form in $n$ dimensions. (Received March 8,1934.) 
177. Professor R. E. Langer: The solutions of the Mathieu equation with a complex variable and at least one parameter large.

The Mathieu differential equation $u^{\prime \prime}+(\Delta-\Omega \cos 2 z) u=0$ is considered over the complex $z$ plane and the asymptotic forms of its solutions are determined for all cases in which the parameters are real and at least one of them is large. When $|\Omega| \geqq|\Delta|$ these forms are subject to the Stokes' phenomenon and this is quantitatively determined. The associated subdivision of the $z$ plane and the forms which describe the solutions in the respective regions depend upon the relative magnitudes of $\Delta$ and $\Omega$. The asymptotic form of the characteristic equation for the parameter values which admit of a periodic solution is obtained, and the form of the characteristic exponent in its dependence upon the parameters is given in each case. (Received March 8, 1934.)

\section{Mr. Nathan Schwid: The asymptotic forms of the Her- mite and Weber functions.}

The Hermite equation, $d^{2} U / d Z^{2}-2 Z d U / d Z+2 K U=0$, and its counterpart under a simple change of variable, the Weber equation, $d^{2} W / d Z^{2}+(2 K+1$ $\left.-Z^{2}\right) W=0$, have been widely discussed. Considerations of the forms of their solutions when the latter are asymptotically dependent upon the parameter $K$ have heretofore been restricted to the case in which $K$ is real, with the variable $Z$, when complex, limited to a finite strip of the $Z$ plane. This paper considers these equations when the parameter is complex and the variable $Z$ is permitted to vary over the entire complex plane. The asymptotic forms of the solutions with respect to large values of $K$ are derived. The results are obtained by utilizing formulas developed by Langer (Transactions of this Society, vol. 34, No. 3, pp. 447-480) for solutions of an ordinary second order differential equation of a certain general structure, a type to which the Weber equation is readily reduced. (Received March 9, 1934.)

179. Professor H. S. Wall: Groups of transformations of continued fractions.

Let $\xi=x_{0}+\left[x_{n} / 1\right]_{1}^{\infty}, \xi_{a}=x_{0}{ }^{a}+\left[x_{n}{ }^{a} / 1\right]_{1}^{\infty}$ be continued fractions with convergents $k_{0} k_{1} k_{2} \cdots$ and $k_{\alpha} k j \cdots k_{\gamma} k_{\alpha+p} k_{\beta+p} \cdots k_{\gamma+p} \cdots$, respectively, where $\alpha \beta \cdots \gamma$ is a permutation of $01 \cdots p-1$. Let $a$ be the substitution which takes $01 \cdots p-1$ into $\alpha \beta \cdots \gamma$, respectively, and let $b$ be another subsitution. The numbers $x_{n}{ }^{a}$ are certain rational functions of some of the $x_{n}$, say $x^{a}=f^{a}(x)$. Then $f^{a}\left(x^{b}\right)=f^{a}\left(f^{b}(x)\right)=f^{a b}(x)=x^{a b}$. For every finite substitution group a simply isomorphic group of birational transformations can be obtained in this way. If $\xi$ converges in an $x$-region, $\xi_{a}$ will converge in the corresponding $x^{a}$-region. Example: If $p=3, a=(012), c=(01)$ then we have $f^{\prime}$ (identity), $f^{a}: x^{a}=-(1+x+y) / x, \quad y^{a}=y z /[(1+x)(1+z)+y], \quad z^{a}=-(1+x+y) /[(1+x)$ $\cdot(1+z)+y] ; f: x=-x /(1+x+y), y=-y /(1+x+y), z^{c}=1 / z ; f^{a}, f^{a c}, f^{c a}$. Here $x=x_{3 n}, \quad y=x_{3 n+1}, \quad z=x_{3 n+2}$. The aggregate of all these transformations $(p=2,3,4, \cdots)$ is an infinite group. (Received March 9, 1934.) 
180. Mr. R. E. Huston: Generalized asymptotic Waring theorems.

For every integral exponent $k \geqq 3$ it is proposed to determine what number $s$ of variables and what sets of positive integral coefficients $a_{1}, \cdots, a_{s}$ insure a solution of the diophantine equation $n=\sum a_{i} h_{i}{ }^{k}, i=1, \cdots, s, h_{i} \geqq 0$, for every integer $n>b$, where $b$ depends only on $k, s$, and the maximum of the $a_{i}$. For $a_{1}=\cdots=a_{s}=1$, the problem reduces to the classical Waring problem in asymptotic form. Here the explicit value $s \geqq(k-2) 2^{k-1}+5$ was established by Hardy and Littlewood. The Hardy-Littlewood mode of attack can be made to yield this same value of $s$ for our more general problem if certain congruential conditions on the $a_{i}$ are satisfied. That these conditions are satisfied by very general sets of coefficients is proved by a sufficiency theorem depending only on the primitivity of the coefficients. An example shows, moreover, that for certain exponents at least no more powerful sufficiency theorem in terms of primitivity of the coefficients alone can be obtained. Two other types of sufficiency theorems are developed and the paper concludes with illustrations of the results obtained for $k=3,4$, and 5. (Received March 9, 1934.)

\section{Mr. B. C. Getchell: Integration of interval functions.}

The norm and sigma limits of functions of subdivisions as developed by Moore and Smith (American Journal of Mathematics, vol. 44 (1922), pp. 101$121)$ are applied to functions with linear intervals as arguments. A necessary and sufficient condition is given for the existence of the norm integral when the sigma integral exists. The class of integrable functions is extended by means of sequences to include generalizations of those classes of point functions which are integrable in the Lebesgue or Stieltjes sense. An integral of the latter type is based upon the property of absolute continuity. (Received March 9, 1934.)

182. Mr. J. W. T. Suckau: On uniform convergence. Preliminary report.

If a sequence of functions converges on a set $S$, then the sequence is uniformly convergent on certain subsets of $S$; e.g., any finite subset. A construction is given which yields all subsets of $S$ on which the convergence is uniform. The object of the paper is twofold. First to investigate the amount of uniform convergence automatically present. It is found that if $S$ is non-denumerable, then there exists a denumerably infinite subset of $S$ on which the convergence is uniform; moreover, an example is given of a sequence of functions which converges on the whole continuum and has the property that on every nondenumerable set the convergence is not uniform. The second object is to restrict the functions and the set $S$ so that uniform convergence is induced on "large" subsets of $S$. It is found that the restricted functions form a very large class, in fact the class of measurable functions. Applications are made to the Lebesgue theory. (Received March 9, 1934.) 
183. Professor H. R. Brahana: Metabelian groups of order $p^{n+m}$ with commutator subgroups of order $p^{m}$.

The metabelian groups considered in this paper are generated by an abelian group of order $p^{n}$ and type $1,1, \cdots$ and $m$ permutable operators of order $p$ from its group of isomorphisms. The number of distinct abstract groups of this kind with centrals of order $p^{n-k}$ is shown to be equal to the number of conjugate sets of matrices $x_{1} M_{1}+x_{2} M_{2}+\cdots+x_{k} M_{k}$, where the $x$ 's are variables and the $M$ 's are $m$-rowed square matrices with elements in a modular field, mod $p$, under two types of transformation: (a) linear homogeneous transformations on the $x$ 's with coefficients in the modular field, and (b) elementary transformations with coefficients in the modular field on the $M$ 's simultaneously. Properties of the groups corresponding to invariant factors of $x_{1} M_{1}+x_{2} M_{2}$, and corresponding to irreducible factors of its determinant are exhibited for $k=2$. These relations obviously generalize to any value of $k$ not greater than $n / 2$. (Received March 9, 1934.)

\section{Dr. H. P. Thielman: Note on the use of fractional integra-} tion for obtaining expansions in squares of Bessel functions.

In this note it is shown that fractional integration of Bessel functions will lead in certain cases to the squares of such functions. Use is then made of this fact to derive new expansions in squares of Bessel functions by fractionally integrating known expansions in first powers of such functions. The derived expansions are valid for the interval in which the known expansions converge uniformly. (Received March 9, 1934.)

\section{Dr. H. P. Thielman: An analogue in Hilbert space of a theorem of Arzela for function space.}

Arzela's theorem is as follows: A necessary and sufficient condition on an infinite set of functions that it contain a uniformly convergent subsequence of continuous functions is that the given set contain an infinite subset which is equibounded and equicontinuous. As an analogue of this we obtain the following result: A necessary and sufficient condition on an infinite set of points in Hilbert space to contain a subsequence which converges uniformly relative to a scale function, which is a point in Hilbert space, is that the infinite set contain an infinite subset which is equibounded and equiconvergent. A set $\left\{x_{i}{ }^{(\alpha)}\right\}$ of points in Hilbert space is defined to be equiconvergent if for every $\epsilon>0$ there exists a positive integer $N$ such that for every choice of $\alpha_{1}{ }^{(i)}, \alpha_{2}{ }^{(i)}$ from the $\alpha$ 's we have $\left.\left.\sum_{i=N}^{\infty}\left[x_{i}{ }^{\left(\alpha_{1}(i)\right.}\right)-x_{i}{ }^{\left(\alpha_{2}(i)\right.}\right)\right]^{2}<\epsilon$. (Received March 9, 1934.)

186. Mr. H. S. Kaltenborn: An extension of Riesz's theorem on linear functional operations.

The most general form of a linear continuous functional operation on a function $f(x)$ continuous on $(a, b)$ has been expressed by F. Riesz (Annales scientifiques de l'Ecole Normale Supérieure, vol. 31 (1914), pp. 9-14) as a Stieltjes 
integral of $f(x)$ with respect to a function of bounded variation. An analogous result is derived in this paper for the case where $f(x)$ has at most discontinuities of the first kind on $(a, b)$. The operation $F[f]$ is expressed in terms of the modified Stieltjes integral considered by B. Dushnik (Thesis, University of Michigan, 1931). If the norm or metric for this class of functions is defined as the least upper bound of $|f(x)|$ on $(a, b)$, then any linear continuous functional operation on $f(x)$ is expressible in the form $T[f]=\int_{a}^{b} f d \psi+\sum_{i=1}^{\infty}\left[f\left(x_{i}\right)\right.$ $\left.-f\left(x_{i}-0\right)\right] \phi\left(x_{i}\right)$, where $x_{1}, x_{2}, x_{3}, \cdots$, denote the points of discontinuity of $f(x), \psi(x)$ and $\phi(x)$ depend only on $T$, and are such that $\psi(x)$ is of bounded variation, and $\phi(x)$ vanishes except at a denumerable set of points and the sum of the absolute values of $\phi(x)$ at these points is finite, and when the integral is of the above mentioned modified Stieltjes type. (Received March 9, 1934.)

187. Professor G. Y. Rainich and Mr. H. E. Vaughan, Jr.: Variation of product integrals.

The question considered is that of change of the value of a line product integral corresponding to a change, not affecting the endpoints, of the path. In index notation the matrices appear as $A_{j k}^{i}$ and the product integral as $\int A_{j r}^{i} d x^{\rho}+\delta_{j}{ }^{i}$. Corresponding to a given path with the endpoints $a$ and $b$ this will be written as $P_{j(a)}^{i(b)}$, omitting the path in the notation. The formula proved is $\Delta P_{j(a)}^{i(b)}=\int_{a}^{b} P_{\rho_{(x)}}^{i(b)} R_{\sigma_{1} \lambda \mu}^{\rho} d x^{\lambda} \Delta x^{\mu} P_{j(a)}^{\sigma(x)} \quad$ where $R_{n_{1} p q}^{m}=\left(\delta / \delta x^{q}\right) A_{n p}^{m}-\left(\delta / \delta x^{p}\right) A_{n q}^{m}$ $+A_{\alpha q}^{m} A_{n p}^{\alpha}=A_{\alpha p}^{m} A_{n q}^{\alpha}$. The proof is made simpler by the use of a special coordinate system. The application to curved spaces where $R$ is the Riemann tensor is obvious. (Received March 9, 1934.)

\section{Professor T. H. Hildebrandt: On bounded linear func- tional operations.}

In this paper, the most general linear bounded functional operation on the vector space of all bounded sequences, convergence being uniform convergence, is determined by the use of an integration process based on the general limits of $\mathrm{E}$. H. Moore. The same procedure yields results in a number of other spaces which are not separable. (Received March 9, 1934.)

189. Professor C. N. Moore: On the relationship between the Abel-Poisson method and that of Cesàro for the summation of multiple series.

In a recent note in the Bollettino della Unione Matematica Italiana (Octo ber, 1933) J. C. Vignaux has considered certain properties of the Abel-Poisson method of summation applied to double series, and he has raised the question as to the relationship between this method and that of Cesàro for such series. In a review of Vignaux's paper in the Zentralblatt (January 27, 1934) E. Kobetliantz has remarked that it would be interesting to have this question settled. It is shown in the present paper that for a multiple series of order $n$ summability $\left(C, \rho_{1}, p_{2}, \cdots, \rho_{n} ; \rho_{i} \geqq 0\right)$ implies summability to the same value 
by the Abel-Poisson method. This is a consequence of certain general theorems on convergence factors in multiple series given in a paper by C. N. Moore (Transactions of this Society, vol. 29 (1927), pp. 227-238). (Received March 9, 1934.)

190. Dr. Gordon Pall: On the order invariants of integral quadratic forms.

Let $A$ denote double the matrix of an integral quadratic form in $s$ variables, of non-zero determinant, the cross-product coefficients not necessarily even; let $s-2 I$ be the signature. For any $k(1 \leqq k \leqq s)$, the g.c.d. of all the principal and doubles of the secondary minor determinants of order $k$ in $A$ is an invariant of the class; this g.c.d. is even if $k$ is odd; denote it by $2 d_{k}$ if $k$ is odd, $d_{k}$ if $k$ is even; define $d_{0}=1$; define $o_{k}$ by $d_{k}{ }^{2} o_{k}=4^{\epsilon} d_{k-1} d_{k+1}\left(\epsilon=1+(-1)^{k}\right)(k=1, \cdots$, $s-1), o_{0}=o_{s}=0$. The $o_{k}$ are integers satisfying (a) $o_{k} \neq 2(\bmod 4) ;(\mathrm{b})$ if any $o_{k}(1 \leqq k \leqq s-1)$ is odd, $o_{k-1} \equiv o_{k+1} \equiv 0(\bmod 16)$; (c) if $o_{1}, o_{3}, \cdots, o_{s-1}$ are odd $\left(s\right.$ even), $(-)^{(s-2 I) / 2} \cdot o_{1} o_{3} \cdots o_{s-1} \equiv 1(\bmod 4) ;(\mathrm{d})$ if all the $o_{k}$ are squares, and $o_{i} \equiv o_{i+1} \equiv 0(\bmod 16)$ for not more than two values $i$, then $s-2 I \not \equiv \pm 3,4(\bmod 8)$. These conditions are necessary and sufficient for a form to exist with the invariants $I, o_{1} \cdots, o_{s-1}$. Further the $k$ th primitive concomitant of $f$ is properly or improperly primitive according as $o_{k}$ is even or odd. H. J. S. Smith's conjecture concerning the invariants of the concomitants is proved. (Received February 9, 1934.) 